\title{
Análisis Bibliométrico de los Artículos Originales Publicados en la Revista Ciencia \& Trabajo: 1999-2015
}

\author{
BIBLIOMETRIC ANALYSIS OF THE ORIGINAL ARTICLES PUBLISHED IN THE JOURNAL CIENCIA \& TRABAJO: \\ 1999-2015
}

\author{
M Ferrer-Villalobos', L Freire-Constante1, P Suasnavas-Bermúdez², P Merino-Salazar³ , A Gómez-García ${ }^{3}$. \\ 1. Profesor Titular de la Ingeniería en Seguridad y Salud Ocupacional. Universidad Internacional SEK. Quito, Ecuador. \\ 2. Decano de la Facultad de Ciencias del Trabajo y del Comportamiento Humano. Universidad Internacional SEK. Quito, Ecuador. \\ 3. Grupo de Investigación sobre Condiciones de Seguridad y Salud en el Trabajo. Universidad Internacional SEK. Quito, Ecuador.
}

\begin{abstract}
RESUMEN
Introducción: La Revista Ciencia y Trabajo (C\&T) es editada por la Fundación Científica y Tecnológica de la Asociación Chilena de Seguridad desde 1999, publica trabajos científicos en temas relacionados con la seguridad e higiene industrial, salud ocupacional, calidad de vida laboral y otras disciplinas asociadas al trabajo y medio ambiente. Objetivo: Analizar la producción científica de los artículos originales publicados en C\&T durante el periodo 1999-2015. Material y Métodos: Estudio bibliométrico descriptivo, longitudinal y retrospectivo. Se analizaron las características metodológicas y técnicas específicas en prevención de riesgos laborales para cada uno de los artículos analizados, asimismo, la visibilidad en SciELO Analytics para los últimos años. Resultados: Se publicaron un total de 430 artículos originales en 52 números con una media de $27 \pm 8,8$ artículos por año y $8 \pm 2,6$ artículos por número. Los años con más artículos fueron 2009, 2010 y 2011. El número de autores por artículo oscila entre 1 a 4 (82\%), en su mayoría de universidades de Latinoamérica, destacando Chile, México y Brasil como los países de mayor contribución. La Psicosociología y Medicina del Trabajo son las temáticas más investigadas respecto a otras técnicas específicas en prevención de riesgos laborales como la Seguridad en el Trabajo y Ergonomía, predominando los estudios transversales de carácter descriptivo-observacional en el idioma español. Conclusiones: Se evidencia la consolidación de la revista a lo largo del tiempo. Por la antigüedad, publicación regular y demás atributos, la Revista C\&T puede plantearse la posibilidad de ser indexada en la base de datos Scopus.
\end{abstract}

(Ferrer-Villalobos M, Freire-Constante L, Suasnavas-Bermúdez P, Merino-Salazar P, Gómez-García A, 2017. Análisis Bibliométrico de los Artículos Originales Publicados en la Revista Ciencia \& Trabajo: 1999-2015. Cienc Trab. May-Ago; 19 [59]: 81-85).

Palabras clave: BIBLIOMETRÍA, REVISTA, ARTÍCULO, BASE DE DATOS, SALUD LABORAL (DeCS).

\section{ABSTRACT}

Background: The journal Science and Work (C\&T) has been published by the Scientific and Technological Foundation of the Chilean Security Association since 1999, it publishes scientific papers on issues related to industrial safety and health, occupational health, quality of work life and other disciplines associated with Work and the environment. Objetive: To analyze the scientific production of the original articles published in C\&T during the period 1999-2015. Materials - Methods: Descriptive, longitudinal and retrospective bibliometric study. We analyzed the specific methodological and technical characteristics in occupational risk prevention for each of the analyzed articles, as well as the visibility in SciELO Analytics for the last years. Results: A total of 430 original articles were published in 52 numbers with an average of $27 \pm 8.8$ articles per year and $8 \pm$ 2.6 articles per number, the years with the most articles were 2009, 2010 and 2011. The number of authors per article ranges from 1 to 4 (82\%), mostly from Latin American universities, Chile, Mexico and Brazil being the countries with the highest contribution. Psychosociology and Occupational Medicine are the most researched topics in relation to other specific techniques in the prevention of occupational hazards such as Occupational Safety and Ergonomics, with cross - sectional descriptive - observational studies predominating in the spanish language. Conclusions: It is evident the consolidation of the journal over time. Due to seniority, regular publication and other attributes, C\&T can consider the possibility of being indexed in the Scopus database.

Keywords: BIBLIOMETRICS, JOURNAL ARTICLE, DATABASE, OCCUPATIONAL HEALTH (MeSH).

\section{INTRODUCCIÓN}

Los estudios bibliométricos permiten conocer la producción científica y la actividad de las revistas dentro de cualquier área de

Correspondencia / Correspondence:

Ph.D. Antonio Ramón Gómez García

Facultad de Ciencias del Trabajo y Comportamiento Humano

Universidad Internacional SEK. Quito, Ecuador

E-mail: antonio.gomez@uisek.edu.ec

Recibido: 20 de Febrero de 2017 / Aceptado: 09 de Abril de 2017 investigación mediante el análisis estadístico de datos. ${ }^{1-2}$ En este sentido, la bibliometría es considerada en la actualidad como una herramienta útil y objetiva ${ }^{1}$, proporcionando información sobre la actividad científica, evolución en el tiempo, productividad de autores, colaboración, impacto y visibilidad de las publicaciones, etc. ${ }^{3}$ De esta manera se ha convertido en una necesidad de las propias revistas para conocer y evaluar su situación. ${ }^{4}$

En el área de la seguridad y salud en el trabajo (SST), la difusión de la producción científica es trascendental, no sólo porque permite difundir los resultados de las investigaciones, sino porque además orienta a la toma de decisiones para la elaboración de políticas y prioridades de investigación en SST. ${ }^{5-7}$ Asimismo, constituye un elemento clave para la generación de 
conocimiento sobre las condiciones de trabajo y la afectación a la salud de los trabajadores basado en evidencias de carácter científico-técnico. ${ }^{8}$

La Revista Ciencia y Trabajo (C\&T; Cienc Trab.) fue creada en 1999 y es editada por la Fundación Científica y Tecnológica de la Asociación Chilena de Seguridad con el apoyo técnico de Environmental Health Perspectives (EHP), Escuela de Medicina de Mount Sinai, Fundación Iberoamericana de Salud Ocupacional, Revista Salud Pública de México y la revista Ciencia \& Saúde Coletiva. En la actualidad se publica cuatrimestralmente de forma impresa (ISSN 0718-0306) y electrónica (ISSN 0718-2449) con acceso libre y a texto completo en las modalidades de editorial, comunicación de casos y artículos (originales editoriales, opinión, revisión, difusión y educación) en temas relacionados con la seguridad e higiene industrial, salud ocupacional, calidad de vida laboral y otras disciplinas asociadas al trabajo y medio ambiente. Se encuentra indexada en las bases de datos Dialnet, EBSCO, Latindex (Catálogo), LILACS, Ulrich's International Periodicals Directory, Psicodoc, e-revistas, IMBIOMED y SciELO. ${ }^{9}$

Dada la importancia de la investigación en SST para mejorar la calidad de vida de la población trabajadora ${ }^{2,7-8}$ y considerando la tradición de la revista C\&T en esta área de conocimiento, el objetivo de este estudio consistió en analizar la producción científica de los artículos originales sobre seguridad y salud en el trabajo publicados en el periodo 1999-2015 mediante el empleo de indicadores bibliométricos, con el fin de analizar su evolución y características, sin entrar en temas relativos a índices de impacto.

\section{MATERIAL Y MÉTODOS}

Estudio bibliométrico descriptivo, longitudinal y retrospectivo de los artículos originales publicados en 52 números desde enero de 1999 a diciembre de 2015, recuperados a través de la versión electrónica de la revista C\&T (http://www.cienciaytrabajo.cl). Como criterio de exclusión se descartaron aquellos documentos de tipo editorial, comunicación, artículos de revisión, etc.; asimismo, no se consideró el año 2001 por tratarse de artículos sobre medicina general (Números 5 y 6).

Para cada artículo se ha distinguido datos relativos al año de publicación, número de la revista, artículos originales por número, primer autor, país de procedencia e institución de afiliación del primer autor, idioma, firmas/artículo y autores con mayor producción.

Simultáneamente, se analizaron las características metodológicas de investigación empleadas en los artículos analizados según la orientación en el tiempo (longitudinales-transversales), finalidad de la investigación (descriptivos-analíticos) y manipulación en el estudio (observacionales-experimentales) ${ }^{10}$; igualmente, se clasificaron por área de estudio en función de las técnicas específicas en prevención de riesgos laborales: Seguridad en el Trabajo, Higiene Industrial, Ergonomía, Psicosociología, Medicina del Trabajo y otros temas (seguridad vial, medio ambiente y otros temas relacionados con la SST). ${ }^{5-11}$

Se consultaron, además, los datos bibliométricos en SciELO Analytics (http://analytics.scielo.org/?journal=0718-2449\&collection=chl):

Tabla 1.

Distribución de los artículos originales en SST publicados en C\&T según año, número y período: 1999-2015.

\begin{tabular}{|c|c|c|c|c|c|c|c|c|c|c|c|c|c|}
\hline Año & Número & Período & - Artículos & $\%$ Anual & Total Artículos & \% Total & Año & Número & Período & $V^{\circ}$ Artículos & $\%$ Anual & Total Artículos & \% Total \\
\hline \multirow[t]{2}{*}{1999} & 1 & Enero-Mayo & 20 & $64,5 \%$ & 31 & $7,2 \%$ & \multirow[t]{4}{*}{2009} & 31 & Enero-Marzo & 8 & $20,5 \%$ & 39 & $9,1 \%$ \\
\hline & 2 & Junio-Diciembre & 11 & $35,5 \%$ & & & & 32 & Abril-Junio & 11 & $28,2 \%$ & & \\
\hline \multirow[t]{2}{*}{2000} & 3 & Enero-Junio & 10 & $62,5 \%$ & \multirow[t]{2}{*}{16} & \multirow[t]{2}{*}{$3,7 \%$} & & 33 & Julio-Septiembre & 10 & $25,6 \%$ & & \\
\hline & 4 & Julio-Diciembre & 6 & $37,5 \%$ & & & & 34 & Octubre-Diciembre & 10 & $25,6 \%$ & & \\
\hline 2001a & $5-6$ & Enero-Diciembre & - & - & - & - & \multirow[t]{4}{*}{2010} & 35 & Enero-Marzo & 9 & $25,7 \%$ & 35 & $8,1 \%$ \\
\hline \multirow[t]{2}{*}{2002} & 7 & Enero-Junio & 7 & $53,8 \%$ & \multirow[t]{2}{*}{13} & \multirow[t]{2}{*}{$3,0 \%$} & & 36 & Abril-Junio & 8 & $22,9 \%$ & & \\
\hline & 8 & Julio-Diciembre & 6 & $46,2 \%$ & & & & 37 & Julio-Septiembre & 8 & $22,9 \%$ & & \\
\hline \multirow[t]{2}{*}{2003} & 9 & Enero-Junio & 4 & $50,0 \%$ & \multirow[t]{2}{*}{8} & \multirow[t]{2}{*}{$1,9 \%$} & & 38 & Octubre-Diciembre & 10 & $28,6 \%$ & & \\
\hline & 10 & Julio-Diciembre & 4 & $50,0 \%$ & & & \multirow[t]{4}{*}{2011} & 39 & Enero-Marzo & 10 & $25,6 \%$ & 39 & $9,1 \%$ \\
\hline \multirow[t]{4}{*}{2004} & 11 & Enero-Marzo & 5 & $20,8 \%$ & \multirow[t]{4}{*}{24} & \multirow[t]{4}{*}{$5,6 \%$} & & 40 & Abril-Junio & 10 & $25,6 \%$ & & \\
\hline & 12 & Abril-Junio & 7 & $29,2 \%$ & & & & 41 & Julio-Septiembre & 10 & $25,6 \%$ & & \\
\hline & 13 & Julio-Septiembre & 6 & $25,0 \%$ & & & & 42 & Octubre-Diciembre & 9 & $23,1 \%$ & & \\
\hline & 14 & Octubre-Diciembre & e 6 & $25,0 \%$ & & & \multirow[t]{4}{*}{$2012 b$} & - & Enero-Marzo & - & - & 22 & $5,1 \%$ \\
\hline \multirow[t]{4}{*}{2005} & 15 & Enero-Marzo & 6 & $23,1 \%$ & \multirow[t]{4}{*}{26} & \multirow[t]{4}{*}{$6,0 \%$} & & 43 & Abril-Junio & 8 & $36,4 \%$ & & \\
\hline & 16 & Abril-Junio & 7 & $26,9 \%$ & & & & 44 & Julio-Septiembre & 7 & $31,8 \%$ & & \\
\hline & 17 & Julio-Septiembre & 6 & $23,1 \%$ & & & & 45 & Octubre-Diciembre & 7 & $31,8 \%$ & & \\
\hline & 18 & Octubre-Diciembre & e 7 & $26,9 \%$ & & & \multirow[t]{3}{*}{2013} & 46 & Enero-Abril & 7 & $26,9 \%$ & 26 & $6,0 \%$ \\
\hline \multirow[t]{4}{*}{2006} & 19 & Enero-Marzo & 8 & $25,8 \%$ & \multirow[t]{4}{*}{31} & \multirow[t]{4}{*}{$7,2 \%$} & & 47 & Mayo-Agosto & 9 & $34,6 \%$ & & \\
\hline & 20 & Abril-Junio & 9 & $29,0 \%$ & & & & 48 & Septiembre-Diciembre & re 10 & $38,5 \%$ & & \\
\hline & 21 & Julio-Septiembre & 7 & $22,6 \%$ & & & 2014 & 49 & Enero-Abril & 9 & $29,0 \%$ & 31 & $7,2 \%$ \\
\hline & 22 & Octubre-Diciembre & e 7 & $22,6 \%$ & & & & 50 & Mayo-Agosto & 10 & $32,3 \%$ & & \\
\hline \multirow[t]{4}{*}{2007} & 23 & Enero-Marzo & 6 & $20,0 \%$ & \multirow[t]{4}{*}{30} & \multirow[t]{4}{*}{$7,0 \%$} & & 51 & Septiembre-Diciembre & e 12 & $38,7 \%$ & & \\
\hline & 24 & Abril-Junio & 7 & $23,3 \%$ & & & 2015 & 52 & Enero-Abril & 13 & $39,4 \%$ & 33 & $7,7 \%$ \\
\hline & 25 & Julio-Septiembre & 8 & $26,7 \%$ & & & & 53 & Mayo-Agosto & 10 & $30,3 \%$ & & \\
\hline & 26 & Octubre-Diciembre & e 9 & $30,0 \%$ & & & & 54 & Septiembre-Diciembre & e 10 & $30,3 \%$ & & \\
\hline 2008 & 27 & Enero-Marzo & 6 & $23,1 \%$ & 26 & $6,0 \%$ & & & & & & & \\
\hline & 28 & Abril-Junio & 6 & $23,1 \%$ & & & & & & & & & \\
\hline & 29 & Julio-Septiembre & 6 & $23,1 \%$ & & & & & & & & & \\
\hline & 30 & Octubre-Diciembre & e 8 & $30,8 \%$ & & & & & & & & & \\
\hline
\end{tabular}

a) Año no incluido en el análisis. b) Período no editado. 
accesos por números de la revista, artículos con mayor número de accesos, revistas citadas en los artículos y citas recibidas de revistas a C\&T desde diciembre de 2013 y diciembre de 2016 para conocer su visibilidad.

Los artículos originales recuperados fueron tabulados en el programa MS Excel 2011 (proceso de calidad y depuración) y analizados con el paquete estadístico SPSS 23. Se realizó un análisis descriptivo empleando las frecuencias, porcentajes, media, desviación estándar, mínimo y máximo para cada una de las variables de estudio.

\section{RESULTADOS}

En la Tabla 1 se presenta la distribución de los artículos originales sobre SST publicados en C\&T por año, número y período. Se ha analizado un total de 430 artículos procedentes de 52 números publicados desde 1999 a 2015 ( $27 \pm 8,8$ artículos/año y $8 \pm 2,6$ artículos/número). No se consideró el año 2001 por tratarse de artículos sobre medicina general (Números 5 y 6), en el período de enero a marzo de 2012 no se editó la revista.

Los años en que se publicaron más artículos fueron el 2009 con 39 artículos (Números 31, 32, 33 y 34), 2010 con 35 (Números 35, 36, 37 y 38) y 39 artículos (Números 39, 40, 41 y 42) en 2011. La revista $C \& T$ ha variado su periodicidad en cuanto a la publicación: semestralmente (1999-2003), trimestralmente (2004-2012) y en la actualidad publica cuatrimestralmente (2013-actualidad). En cuanto al lugar de procedencia del primer autor, predominan los países de Latinoamérica con el 88,1\%; Chile ( $\mathrm{n}=244 ; 56,7 \%$ ), México $(n=54 ; 12,6 \%)$ y Brasil $(n=29 ; 6,7 \%)$, seguido de países del continente europeo con el 7,0\%. Destaca España con 22 (5,1\%) artículos originales publicados. En la Tabla 2 se muestra la distribución porcentual del número de artículos por país del primer autor.

Según afiliación del primer autor, las universidades concentran más del 50\% de los artículos publicados, seguidos de la Asociación Chilena de Seguridad -Hospital del Trabajador-, empresas

Tabla 2.

Porcentaje de contribución de los paises en la Revista Ciencia y Trabajo entre 1999-2015.

$\begin{array}{llcc}\text { Continente } & \text { País } & \text { No Artículos } & \% \text { Total } \\ \text { Asia }(n=2) & \text { Israel } & 1 & 0,2 \% \\ \text { Europa }(n=30) & \text { Japón } & 1 & 0,2 \% \\ & \text { Alemania } & 1 & 0,2 \% \\ & \text { Austria } & 1 & 0,2 \% \\ & \text { Bélgica } & 1 & 0,2 \% \\ & \text { España } & 22 & 5,1 \% \\ & \text { Finlandia } & 1 & 0,2 \% \\ \text { Latinoamérica }(n=379) & \text { Holanda } & 1 & 0,2 \% \\ & \text { Portugal } & 3 & 0,7 \% \\ & \text { Argentina } & 7 & 1,6 \% \\ & \text { Brasil } & 29 & 6,7 \% \\ & \text { Chile } & 244 & 56,7 \% \\ & \text { Colombia } & 12 & 2,8 \% \\ & \text { Costa Rica } & 2 & 0,5 \% \\ \text { Sin especificar } & \text { Cuba } & 1 & 0,2 \% \\ & \text { Ecuador } & 5 & 1,2 \% \\ \text { Norteamérica }(n=10) & \text { México } & 54 & 12,6 \% \\ & \text { Perú } & 11 & 2,6 \% \\ & \text { Uruguay } & 1 & 0,2 \% \\ & \text { Venezuela } & 13 & 3,0 \% \\ & \text { Canadá } & 2 & 0,5 \% \\ & \text { Estados Unidos } & 8 & 1,9 \% \\ & & 9 & 2,1 \%\end{array}$

privadas y hospitales-clínicas, destacando la escasa representación de los organismos públicos.

Las instituciones de educación superior de mayor producción son la Universidad de Chile, Universidad de Guadalajara, Universidad de Concepción, Universidad Católica San Pablo y Universidad Nacional Autónoma de México, principalmente.

Con respecto al idioma empleado, se observa un predomino de los artículos editados en español (96,3\%) respecto al portugués $(2,1 \%)$ e inglés $(1,6 \%)$, apareciendo estos dos últimos idiomas a partir de 2011 (Número 40).

En relación al número de autores por artículo, los 430 artículos originales fueron firmados por 1.253 autores, $3 \pm 1,7$ autores/ artículo. El 47,7\% $(\mathrm{n}=205)$ de los artículos están firmados por 1 a 2 autores, el $34,2 \%(n=147) 3$ a 4 y por 5 a 6 autores el $14,0 \%$ ( $\mathrm{n}=60$ ) de los artículos, principalmente.

Los investigadores (primer autor) con más trabajos originales publicados fueron: Arias W.L. con 8 artículos, Córdova V. con 7, Trucco M. con 6, Lasso J. y Rebolledo P. con 5 y con 4 artículos Blamey X., Contreras C.A., Fica G., Olivares V.E. y Vera A. como los mayores productores en C\&T.

En cuanto a las técnicas específicas en prevención de riesgos laborales más investigadas en los artículos originales destacan, en primer lugar: la Psicosociología ( $\mathrm{n}=144 ; 33,5 \%)$ y Medicina del Trabajo ( $n=143 ; 33,3 \%$ ), seguidos de la Seguridad en el Trabajo y Ergonomía ( $\mathrm{n}=47 ; 10,9 \%$ ) y otros temas de estudio relacionados con la seguridad y salud en el trabajo $(\mathrm{n}=33 ; 7,7 \%) \mathrm{y}$, por último, la Higiene Industrial ( $\mathrm{n}=16 ; 3,7 \%$ ), Fig. 1 A-B. Predominando los estudios transversales $(\mathrm{n}=373)$, de carácter descriptivo $(\mathrm{n}=314)$ y observacional $(n=360)$.

\section{Figura 1.}

Evolución de los artículos publicados según técnicas en prevención de riesgos laborales: 1999-2015.
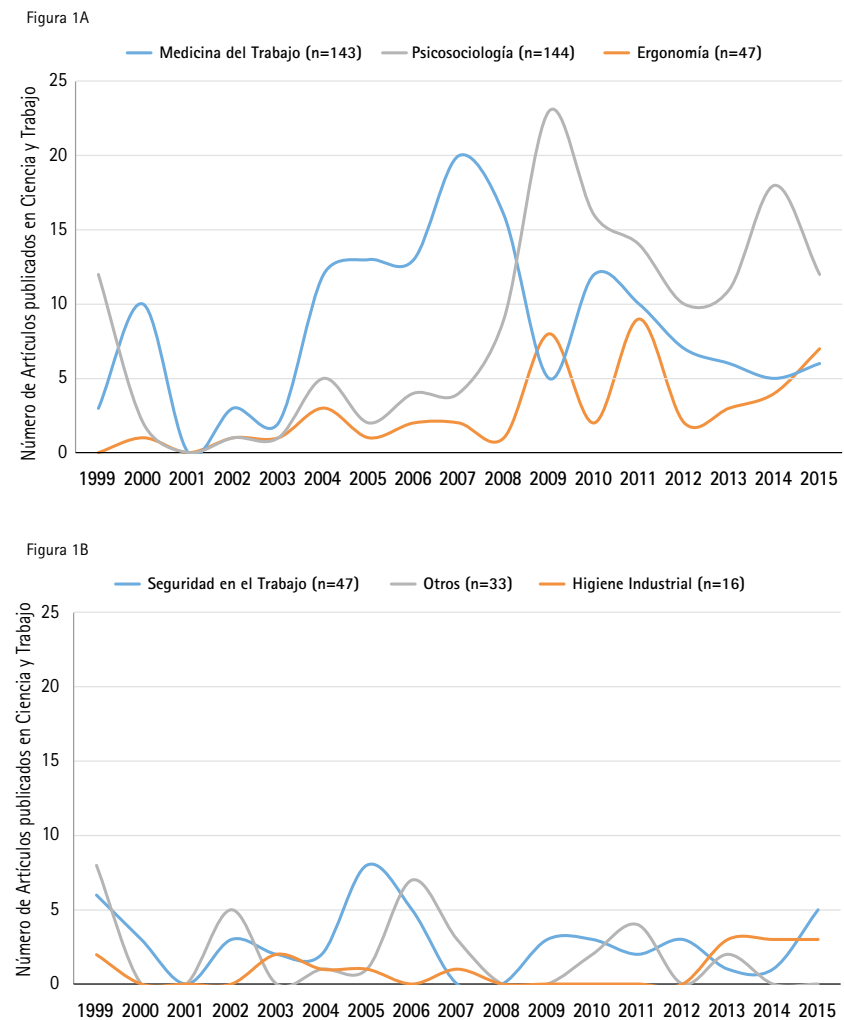
Tabla 3.

Principales artículos originales en SST visitados en C\&T: SciELO Analytics 2013-2015.

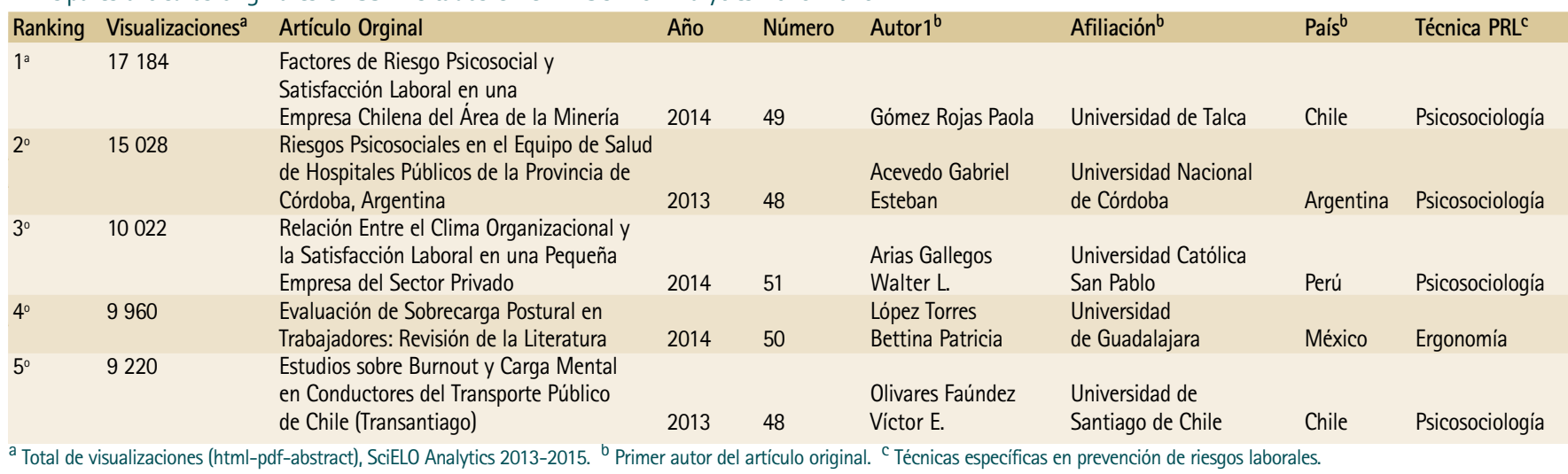

Finalmente, a partir de la información de SciELO Analytics (Chile) para el período comprendido entre noviembre de 2013 a diciembre de 2015, el número de accesos a los documentos de la revista C\&T fue de $329.419(\mathrm{html}=258.073$; pdf $=56.141$; abstract $=15.205)$, siendo los números más visualizados el № 48 , con 62.325 accesos, el No 49 con 47.818 , № 47 con 39.055 , el № 51 con 34.538 y con 34.390 accesos el No 46. En la Tabla 3 se presentan los principales artículos originales en SST más visitados.

Los artículos publicados en Ciencia y Trabajo han citado un total de 3.307 documentos procedentes de 2.421 revistas científicas (incluida C\&T; 77), siendo las principales revistas más citadas: Applied Ergonomics con 28 citas, Occupational and Environmental Medicine con 23, Ergonomics con 19, Medicina y Seguridad del Trabajo con 19 y con 18 citas la Journal of Occupational Rehabilitation.

En cuanto a las citas recibidas en C\&T por otras revistas científicas destaca la Revista de Medicina y Seguridad del Trabajo editada por la Escuela Nacional de Medicina del Trabajo de España con 10 citas, la Revista Científica Salud Uninorte de la Universidad del Norte de Colombia con 9, Revista Sociológica de la Universidad Autónoma Metropolitana de México con 6 y con 5 citas recibidas la Revista Ciencia y Enfermería de la Universidad de Concepción de Chile, Revista de Psicología (Fractal) de la Universidade Federal Fluminense de Brasil y la revista Universitas Psychologica de la Pontificia Universidad Javeriana de Colombia.

\section{DISCUSIÓN}

El presente estudio caracteriza la evolución y producción de los artículos originales publicados en la revista C\&T durante 16 años, mediante el análisis de indicadores bibliométricos.

La revista C\&T se encuentra indexada en diferentes bases de datos del área salud (IMBIOMED, LILACS y EBSCO), la psicología (PSICODOC) y otras áreas de conocimiento (SciELO, Latindex y Dialnet) de América Latina, el Caribe, España y Portugal, permitiendo su visibilidad.

Una de las características de la revista C\&T es la procedencia del primer autor, ya que los artículos originales provienen principalmente de países de Latinoamérica $(88 \%)$ y en su mayoría de universidades. ${ }^{14}$ Animamos a los investigadores de otras áreas geográficas a publicar sus estudios en esta revista.

El número de autores por artículo original es similar a otros estudios bibliométricos de características similares ${ }^{2-12}, 1$ a 4 autores (82\%). Existe una relación del número de firmantes de un trabajo con su aceptación para ser publicados en una revista, encontrándose un mayor índice de aceptación de los trabajos con el mayor número de firmas por artículo, manifestando la colaboración en la investigación multidisciplinar como universal y esencial. ${ }^{13}$

Otro hallazgo muy relevante es que la Psicosociología destaca como la técnica preventiva más investigada, predominando los estudios transversales, de carácter descriptivo y observacional. ${ }^{10}$ Este hecho puede ser debido a dos aspectos; el primero, en las últimas décadas ha aumentado el interés por la investigación en esta área de conocimiento ${ }^{15} \mathrm{y}$, en segundo lugar, si bien el empleo de los análisis estadísticos está determinado por el objeto de estudio investigado, consideramos oportuno que los futuros autores realicen estudios con métodos estadísticos más complejos que aumenten la calidad de su resultados y, por tanto, el prestigio de la revista.

En cuanto a las principales revistas que citan a C\&T, los primeros puestos del ranking se encuentran ocupados por revista de prestigio a nivel internacional, como es el caso de la Revista de Medicina y Seguridad del Trabajo. Igualmente, citas incluidas en los artículos publicados en C\&T: Applied Ergonomics y Occupational and Environmental Medicine, principalmente.

\section{CONCLUSIÓN}

De los resultados de este estudio podemos concluir que la Revista Ciencia y Trabajo (Cienc Trab.) -por su antigüedad, calidad en el proceso editorial, publicación periódica y regular, relevancia en el área de seguridad y salud en el trabajo y demás atributos-, puede plantearse la posibilidad de ser indexada en la base de datos Scopus, al igual que otras revistas de alto prestigio como: Scandinavian Journal of Work; Environment and Health, Occupational and Environmental Medicine; Revista Cubana de Higiene; Epidemiologia y Revista de Saude Publica. 


\section{REFERENCES}

1. Vicente Herrero MT, López González AA, Ramírez Iñíguez de la Torre MV, Capdevila García LM, Terradillos García MJ, Aguilar Jiménez E. Fundamentos del análisis bibliométrico y su aplicación al campo de la salud laboral. Arch Prev Riesgos Labor. 2014; 17(3):154-155.

2. Velázquez López D, Robledillo Colmenares A, Mangas Gallardo I, Veiga Cabo J, Maqueda Blasco J. Análisis bibliométrico de la revista Medicina y Seguridad del Trabajo durante el periodo 2007-2012. Med Segur Trab. 2013; 59(233):383392.

3. Bordons $M$, Zulueta MA. Evaluación de la actividad cientifica a través de indicadores bibliométricos. Rev Esp Cardiol. 1999; 52(10):790-800.

4. Ferriols $R$, Santos $B$, Artacho $S$, Clopés $A$, Guerrero MD, Martínez MJ, Ordovás JP, Otero MJ. Análisis bibliométrico de la revista Farmacia Hospitalaria (20012006). Farm Hosp. 2006; 31:141-149.

5. Estrada M, Sánchez Tovar L, Gómez W. Aproximación al estudio de las tendencias e impacto de la producción científica en salud ocupacional: Estudio de una publicación periódica. Salud Trab. 2006; 14(2):69-79.

6. Benavides $F$, Maqueda J. La investigación en seguridad y salud en el trabajo: ¿Un paso adelante? Arch Prev Riesgos Labor. 2003; 6:55-56.
7. Benavides, $F$, et al. Prioridades de investigación en salud laboral en España. Med Segur Trab. 2008; 54(211):25-27.

8. Gómez AR, Suasnavas PR, Vilaret A, Silva MG, Russo M. Propuesta metodológica para el establecimiento de líneas de investigación en seguridad y salud en el trabajo. INNOVA Res J. 2016; 1(9):13-25.

9. Olivares VE. Revista C\&T desde 1999. Cienc trab. 2013; 15(48):A33.

10. Mirón JA, Sardón A, Iglesias de Sena H. Metodología de investigación en Salud Laboral. Med Segur Trab. 2010; 56(221):347-365.

11. Cortés JM. Técnicas de prevención de riesgos laborales: Seguridad e Higiene del Trabajo., Madrid: Tebar; 2006.

12. Sanz J. Internet en la recuperación de las referencias bibliográficas de las revistas de Salud Pública, indizadas en la red SciELO-España, en el periodo 2000 a 2004 [tesis]. Alicante: Universidad de Alicante; 2006.

13. Greene M. The demise of the lone author. Nature. 2007; 450(7173):1165.

14. Calvert J, Patel P. University-industry research collaborations in the UK: bibliometric trends. Sci Publ Policy. 2003; 30(2):85-96.

15. Salazar A. Salud, burnout y estrés en ámbitos laborales. Una revisión sistemática. Psicología y Salud. 2015; 25(2):147-155. 